\title{
eHealthcare: Was bedeutet die Integration der Informations- und Kommunikations- technologien für die Zukunft der Ärzteschaft?
}

Das Gesundheitswesen muss und wird sich verändern. Als tragender Teil des Gesundheitswesens werden sich auch die Ärztinnen und Ärzte mitverändern müssen. Wir haben dabei lediglich die Wahl, ob wir die anstehenden Veränderungen passiv über uns ergehen lassen oder ob wir die Entwicklungen aktiv mitgestalten wollen.

Die Veränderungen im Gesundheitswesen werden mit der Integration von Informationsund Kommunikationstechnologien einhergehen, es werden daraus völlig neue Möglichkeiten entstehen. Es zeichnen sich dabei neue Handlungsfelder im Gesundheitswesen ab, welche Auswirkungen auf das Selbstverständnis und die Rollen aller Beteiligten haben werden.

Auf die Bürger, Konsumenten oder Patienten kommen heute noch schwer vorstellbare Neuerungen zu. Die sinnvolle, aber auch sichere Nutzung des zur Verfügung stehenden technologischen Potentials muss insbesondere Bezug nehmen auf die qualitativen und Beziehungsbedürfnisse der Bevölkerung. Dafür brauchen wir eine Erneuerung der Allianz zwischen Patient und Arzt, welche auf Vertrauen und gegenseitiger Wertschätzung gründet.

Für die Ärzteschaft ermöglichen durch Informatikmittel unterstützte Versorgungsnetzwerke neue Formen der Organisation, aber auch der Autonomie. Diese Mittel eröffnen wiederum neue Qualitäten des Wissensmanagements, mit unmittelbarer Auswirkung auf den klinischen Alltag. Die informatisch unterstützte Arbeit am und mit dem Patienten wird die evidenzbasierte Medizin und die Aus-, Weiter- und Fortbildung fruchtbar miteinander verbinden.

Diese Entwicklungen und die sich daraus eröffnenden Berufsfelder zwingen die Ärzte, ihr berufliches Selbstverständnis neu zu definieren. Mit Blick auf die Zukunft müssen wir auf unsere historischen Wurzeln und die damit verbundenen ethischen Ideale und Werthaltungen zurückgreifen. Die Verbindung von Altem mit Neuem wird allen eine bessere Medizin erschliessen.

Dr. med. Martin D. Denz, Chairman Kongress eHealthCare.ch, Leiter Departement eHealthcare FMH

\section{eHealthCare.ch 03}

Neue Technologien und IT in Medizin und Gesundheitswesen

Konferenz und Fachmesse

Universität Zürich-Irchel, 16./17. Oktober 2003

Schwerpunkt: Der Patient im Informationszeitalter

www.eHealthCare.ch

Leading Mediapartner: EMH Schweizerischer Ärzteverlag AG 